\title{
ON LATTICE COMPLEMENTS
}

\section{by ROBERT BUMCROT}

(Received 6 January, 1964)

Let $(L, \leqq)$ be a distributive lattice with first element 0 and last element 1 . If $a, b$ in $L$ have complements, then these must be unique, and the De Morgan laws provide complements for $a \vee b$ and $a \wedge b$. We show that the converse statement holds under weaker conditions.

THEOREM 1. If $(L, \leqq)$ is a modular lattice with 0 and 1 and if $a, b$ in $L$ are such that $a \vee b$ and $a \wedge b$ have (not necessarily unique) complements, then $a$ and $b$ have complements.

Proof. We indicate by $={ }^{*}$ the steps at which the modular condition is used. Choosing any complements $(a \vee b)^{\prime}$ and $(a \wedge b)^{\prime}$ of $a \vee b$ and $a \wedge b$ respectively, let

Then

$$
x=(a \vee b)^{\prime} \vee\left[(a \wedge b)^{\prime} \wedge b\right] .
$$

$$
\begin{aligned}
a \vee x & =a \vee(a \wedge b) \vee(a \vee b)^{\prime} \vee\left[(a \wedge b)^{\prime} \wedge b\right] \\
& ={ }^{*} a \vee(a \vee b)^{\prime} \vee\left\{\left[(a \wedge b) \vee(a \wedge b)^{\prime}\right] \wedge b\right\}=a \vee(a \vee b)^{\prime} \vee b=1,
\end{aligned}
$$

and

$$
\begin{aligned}
a \wedge x & =a \wedge(a \vee b) \wedge\left\{(a \vee b)^{\prime} \vee\left[(a \wedge b)^{\prime} \wedge b\right]\right\} \\
& ={ }^{*} a \wedge\left\{0 \vee\left[(a \wedge b)^{\prime} \wedge b\right]\right\}=a \wedge(a \wedge b)^{\prime} \wedge b=0,
\end{aligned}
$$

so $x$ is a complement of $a$. Similarly $y=(a \vee b)^{\prime} \vee\left[(a \wedge b)^{\prime} \wedge a\right]$ is a complement of $b$.

This provides an extension of the De Morgan laws to modular lattices.

THEOREM 2. If $(L, \leqq)$ is a modular lattice with 0 and 1, if $a, b$ in $L$ have unique complements $a^{\prime}, b^{\prime}$ respectively, and if $a \vee b$ and $a \wedge b$ have complements, then $a^{\prime} \vee b^{\prime}$ is a complement of $a \wedge b$ and $a^{\prime} \wedge b^{\prime}$ is a complement of $a \vee b$.

Proof. Form $x$ and $y$ as in the above proof. By dualization, $\bar{x}=(a \wedge b)^{\prime} \wedge\left[(a \vee b)^{\prime} \vee b\right]$ and $\bar{y}=(a \wedge b)^{\prime} \wedge\left[(a \vee b)^{\prime} \vee a\right]$ are also complements of $a$ and $b$ respectively. Then since $a^{\prime}$ and $b^{\prime}$ are unique, $a^{\prime}=x=\bar{x}$ and $b^{\prime}=y=\bar{y}$. Now

and

$$
\begin{aligned}
a^{\prime} \vee b^{\prime} \vee(a \wedge b) & =x \vee y \vee(a \wedge b) \\
& =(a \vee b)^{\prime} \vee\left[(a \wedge b)^{\prime} \wedge b\right] \vee\left[(a \wedge b)^{\prime} \wedge a\right] \vee(a \wedge b) \\
& ={ }^{*}(a \vee b)^{\prime} \vee\left\{b \wedge\left[(a \wedge b)^{\prime} \vee(a \wedge b)\right]\right\} \vee\left\{a \wedge\left[(a \wedge b)^{\prime} \vee(a \wedge b)\right]\right\} \\
& =(a \vee b)^{\prime} \vee a \vee b=1,
\end{aligned}
$$

$$
\begin{aligned}
\left(a^{\prime} \vee b^{\prime}\right) \wedge(a \wedge b) & =(\bar{x} \vee \bar{y}) \wedge(a \wedge b) \\
& =\left\{\left[(a \wedge b)^{\prime} \wedge\left[(a \vee b)^{\prime} \vee b\right]\right] \vee\left[(a \wedge b)^{\prime} \wedge\left[(a \vee b)^{\prime} \vee a\right]\right]\right\} \wedge(a \wedge b) \\
& ={ }^{*}(a \wedge b)^{\prime} \wedge\left\{(a \vee b)^{\prime} \vee b \vee\left[(a \wedge b)^{\prime} \wedge\left[(a \vee b)^{\prime} \vee a\right]\right]\right\} \wedge(a \wedge b)=0,
\end{aligned}
$$

so $a^{\prime} \vee b^{\prime}$ is a complement of $a \wedge b$. Dually $a^{\prime} \wedge b^{\prime}$ is a complement of $a \vee b$.

Figure 1 shows a modular lattice of order seven which contains uniquely complemented elements $a$ and $b$ such that $a \vee b$ has no complement. Contrary to Exercise 4, p. 153 of [1], 
there are no lattices of order six or less, modular or otherwise, in which the complemented elements fail to form a sublattice.

If $(L, \leqq)$ is a complemented modular lattice, then, by a theorem of J. von Neumann $[1, p .124]$, the uniquely complemented elements constitute the center of the lattice, which in any lattice with 0 and 1 is a sublattice $[1$, p. 27]. But $[1$, p. 120] in a complemented modular

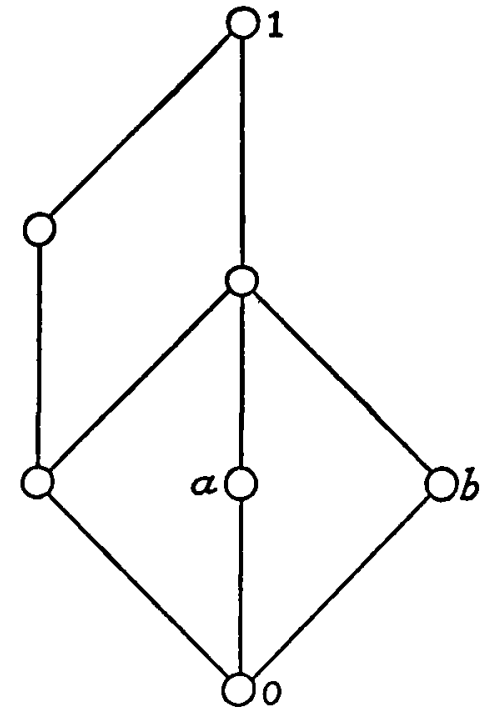

FIG. 1.

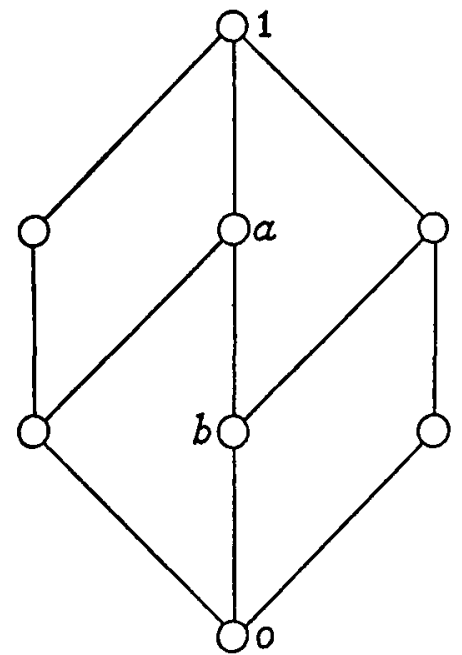

FIG. 2.

lattice every central element is neutral, and hence $[1$, p. 28] distributes with every pair of elements of the lattice. Thus in a complemented modular lattice those elements which have unique complements form a Boolean algebra, and hence satisfy the De Morgan laws. Some questions in this direction remain unanswered. For example, if $(L, \leqq)$ is modular, if $a$ and $b$ have unique complements in $L$, and if $a \vee b$ and $a \wedge b$ have complements, are these complements necessarily unique?

Finally, Dilworth [2] has shown, without giving an example, the existence of non-distributive lattices in which each element has a unique complement. Such lattices are non-modular by what we have said above. It seems doubtful that the De Morgan laws would hold in all such lattices. Figure 2 shows a complemented, though not uniquely complemented, nonmodular lattice containing elements $a$ and $b$ such that $a, b, a \vee b$ and $a \wedge b$ have unique complements, but both De Morgan laws fail.

\section{REFERENCES}

1. G. Birkhoff, Lattice theory (American Mathematical Society Colloquium Publications, Vol. 25; 2nd edition, 1948).

2. R. P. Dilworth, Lattices with unique complements, Trans. Amer. Math. Soc. 57 (1945), 123-154.

OHIO State University

COlumbus, OHIO 